\title{
BDS Common-View Method Based on CGGTTS V2E Standard
}

\author{
$\mathrm{Xu} \mathrm{Liu}^{1,2}$, Dangli Zhao ${ }^{1}$ and Junliang Liu ${ }^{1}$ \\ ${ }^{1}$ National Time Service Centre, Chinese Academy of Sciences, Xi'an 710600, China \\ ${ }^{2}$ Graduate University of Chinese Academy of Sciences, Beijing 100039, China
}

\begin{abstract}
CCTF published CGGTTS for Common GNSS Generic Time Transfer Standard that is CGGTTS V2E. To support the standard in the BDS common-view, the paper refers to the standard and simulates corresponding algorithm in the standard realizing BDS common-view method based on CGGTTS V2E. The result show that the accuracy is about 1ns based on BDS in processing algorithm that is mentioned in the standard.
\end{abstract}

\section{Keywords-BDS; CGGTTS V2E; common-view}

\section{INTRODUCTION}

The common-view method is a main form that is used for time transfer. With the development of BDS, the common-view method based on BDS is becoming increasingly important in many fields of China. In addition, the CGGTTS V01 [1] only supports GPS and the CGGTTS V02 only covers GPS and GLONASS [2], but the CGGTTS V2E [3] provides extension for all GNSS system in development. Meanwhile, the CGGTTS V2E is fully compatible with the previous versions GGTTS V01 and CGGTTS V02 for the data progressing algorithm and data format. Taken together, common-view based BDS can be implemented and is available. Therefore, the paper simulates the computation algorithm raised by CGGTTS V2E providing supports to the development of time transfer receiver based on BDS.

\section{THE PRINCIPLE OF COMMON-VIEW}

The common-view is one of the ways being used for remote time transfer. As FIGURE 1, assume that user A and user B are located at two points on the earth. A and B observe the same satellite at the same time. Suppose the local time of A is $\tau_{\mathrm{A}}$ and the local time of $\mathrm{B}$ is $\tau_{\mathrm{B}}$, the satellite clock is $\tau_{\mathrm{S}}$. The time delay of signal propagation is $\Delta \tau_{\mathrm{A}}$ and $\Delta \tau_{\mathrm{B}}$ respectively. So the offset between the local time of user $A$ and the satellite clock is $\tau_{\mathrm{AS}}$ and the offset between the local time of user $\mathrm{B}$ and the satellite clock is $\tau_{\mathrm{BS}}[1]$. The $\tau_{\mathrm{AS}}$ and $\tau_{\mathrm{BS}}$ will be obtained as:

$$
\begin{gathered}
\tau_{\mathrm{AS}}=\tau_{\mathrm{A}}-\tau_{\mathrm{S}}-\Delta \tau_{\mathrm{A}} \\
\tau_{\mathrm{BS}}=\tau_{\mathrm{B}}-\tau_{\mathrm{S}}-\Delta \tau_{\mathrm{B}}
\end{gathered}
$$

The offset between local time of user A and local time of user
$\mathrm{B}$ is $\tau_{\mathrm{AB}}$ that will be obtained as:

$$
\begin{aligned}
\tau_{\mathrm{AB}} & =\tau_{\mathrm{AS}}-\tau_{\mathrm{BS}} \\
& =\left(\tau_{\mathrm{A}}-\tau_{\mathrm{B}}\right)-\left(\Delta \tau_{\mathrm{A}}-\Delta \tau_{\mathrm{B}}\right)
\end{aligned}
$$

Common-View method can remove the satellite clock, and partly eliminate the time delay of signal propagation.



FIGURE I. THE PRINCIPLE OF COMMON-VIEW

\section{DAta Processing Based CGgtTs V2E}

The data to be used are the pseudorange measurements collected during 13 minutes. The original GGTTS directive asked for 1-second measurements grouped into blocks of 15 seconds. Then Defraigne et al. proposed a collection method that is a 30 -second sampling rate. These authors verified that the differences between CGGTTS results based on either method is less than $0.1 \mathrm{~ns}$. The sampling rate of observation raw-data in this paper is a 30 -seconds. In other words, give a total of 26 data per 13-minute track.

First, the pseudorange measurements collected are corrected for their frequency-dependent hardware delay as:

$$
\bar{P}_{i}=P_{i}-c\left(t_{\mathrm{INTDLY}}\left(f_{i}\right)+t_{\mathrm{CABDLY}}-t_{\mathrm{REFDLY}}\right)
$$

where $P_{i}$ is pseudorange measurement on the frequency $f_{i}$, $c$ is the velocity of the light in the vacuum, $t_{\text {INTDLY }}\left(f_{i}\right)$ is the electric delay of the GNSS signal inside the antenna and the receiver, $t_{\mathrm{CABDLY}}$ is the signal group delay inside the antenna cable, $t_{\text {REFDLY }}$ is the offset between the receiver internal clock and the local clock.

Second, for B1I signal frequency users the offset between 
local clock and the satellite clock that is REFSV will be obtained as:

$$
\begin{aligned}
t_{\text {clock }}-t_{\text {sat }} & =\frac{1}{c}\left[\overline{P_{1}}-\left\|\overrightarrow{x_{\text {sat }}}-\overrightarrow{x_{\text {rec }, 1}}\right\|-S\right]+t_{\text {rel }} \\
& -t_{\text {iono }, 1}-t_{\text {tropo }}-t_{\mathrm{GD}}
\end{aligned}
$$

where $\overrightarrow{x_{\text {sat }}}$ is the satellite position at the emission time, $\overrightarrow{x_{\text {rec, }, 1}}$ is the position of the phase center of antenna for frequency $f_{1}$ that is B1I signal frequency for BDS, $S$ is the Sagnac correction, $\Delta t_{\text {rel }}$ is the relativistic clock correction, $\Delta t_{\text {iono,1 }}$ is the propagation delay caused by ionospheric refraction, $\Delta t_{\text {tropo }}$ is the propagation delay caused by tropospheric refraction, $t_{\mathrm{GD}}$ is the broadcast group delay.

The $\Delta t_{\text {iono, } 1}$ will be obtained by Klobuchar model [4] and the $\Delta t_{\text {tropo }}$ will be obtained by Saatamoninen model and GPT/GMF model [5].

The $\overrightarrow{x_{\text {sat }}}$ will be obtained based on ICD file on BDS [6]. Meanwhile, the Sagnac is corrected by a rotation matrix [7]. Assume that the satellite position is $\left(x_{\mathrm{k}}, y_{\mathrm{k}}, z_{\mathrm{k}}\right)$ in the ECEF at the emission time, and $\left(x^{(\mathrm{s})}, y^{(\mathrm{s})}, z^{(\mathrm{s})}\right)$ in the ECEF at the reception time. The Sagnac correction will be obtained as:

$$
\left[\begin{array}{c}
x^{(\mathrm{s})} \\
y^{\mathrm{s}} \\
z^{(\mathrm{s})}
\end{array}\right]=R\left(\dot{\Omega}_{e} \tau\right) \cdot\left[\begin{array}{c}
x_{\mathrm{k}} \\
y_{\mathrm{k}} \\
z_{\mathrm{k}}
\end{array}\right]
$$

where the $R$ is a rotation matrix:

$$
R(\varphi)=\left[\begin{array}{ccc}
\cos \varphi & \sin \varphi & 0 \\
-\sin \varphi & \cos \varphi & 0 \\
0 & 0 & 1
\end{array}\right]
$$

The $\Delta t_{\text {rel }}$ will be obtained as $[6,7]$ :

$$
\Delta t_{\mathrm{k}}=-\frac{2}{\mathrm{c}^{2}} \cdot \mathrm{e} \cdot \sqrt{\mathrm{a} \mu} \cdot \sin E_{\mathrm{k}}
$$

where $\mathrm{a}$ is the semimajor axis, e is the orbit eccentricity of the satellite orbit and $E_{\mathrm{k}}$ is the eccentric anomaly of the satellite, an angle relate to the position of the satellite on its orbit.

Third, for each of the 26 epochs, the broadcast satellite clock offset with respect to the reference time scale of the BDS is then added to the result of (5), which gives:

$$
t_{\text {clock }}-t_{\text {ref }}=\left(t_{\text {clock }}-t_{\text {sat }}\right)+\Delta t_{\text {sat }}
$$

where the $\Delta t_{\text {sat }}$ will be obtained as:

$$
\Delta t_{\mathrm{sv}}=a_{0}+a_{1}\left(t-t_{\mathrm{oc}}\right)+a_{2}\left(t-t_{\mathrm{oc}}\right)^{2}
$$

where $a_{0}, a_{1}, a_{2}$ are the polynomial coefficients related to satellite clock, $t_{\mathrm{oc}}$ is the clock data reference time in seconds.

According to (5), the computation algorithm flow diagram as FIGURE 2



FIGURE II. THE COMPUTATION ALGORITHM FLOW DIAGRAM THE TESTING PLATFORM

The testing platform is comprised of two receivers using time and frequency transfer, two $10 \mathrm{MHz}$ references and two PPS signals and two antennas. The $10 \mathrm{MHz}$ reference and PPS signal originating from a local clock. The receivers supports standard inputs for time and frequency as well as incorporates a calibration circuit to measure and compensate for the delay between the PPS input and the internal time reference and outputs observation data and navigation data in RINEX format The system diagram as FIGURE 3. The receiver at master station is marked as MASTER, and the receiver at remote station is marked as REMOTE.

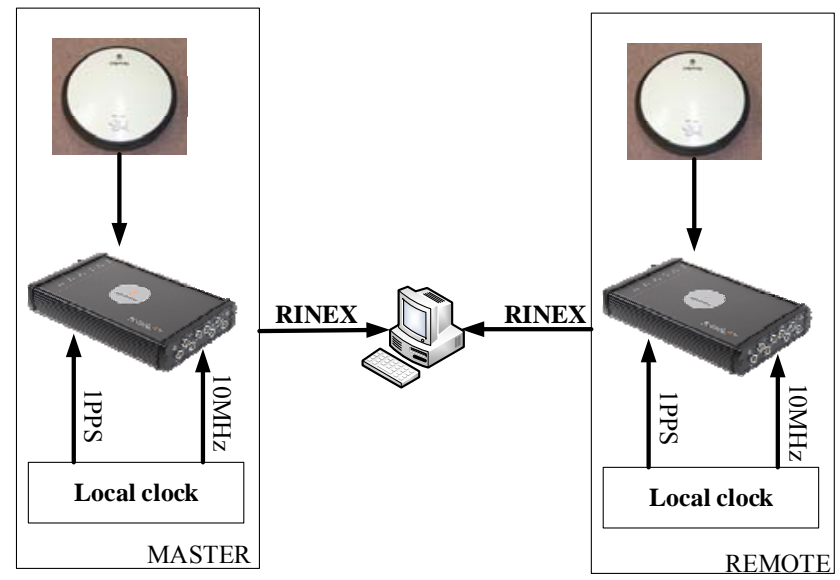

FIGURE III. SYSTEM DIAGRAM ALGORITHM SIMULATION 
In this section, the paper will show some simulation results. There results contains three parts. First, the offset between the local clock and the satellite clock are represented REFSV. Second, the offset between the local clock and the reference time scale of the BDS are represented REFSYS. Third, the offset between the local clock at MASTR station and the local clock at REMOTE station.

To PRN13 of BDS as example, the paper demonstrates the simulation results including the REFSV, the REFSYS and the offset between the local clock at MASTR station and the local clock at REMOTE station. The results as in FIGURE 4 6:
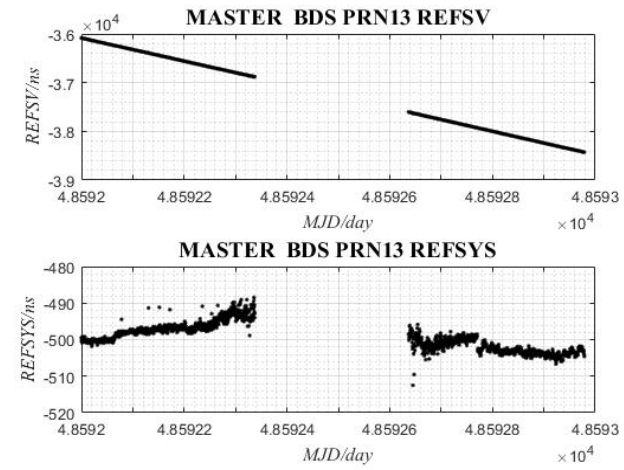

FIGURE IV. THE REFSV AND REFSYS AT MASTER STATION BASED PRN13 OF BDS
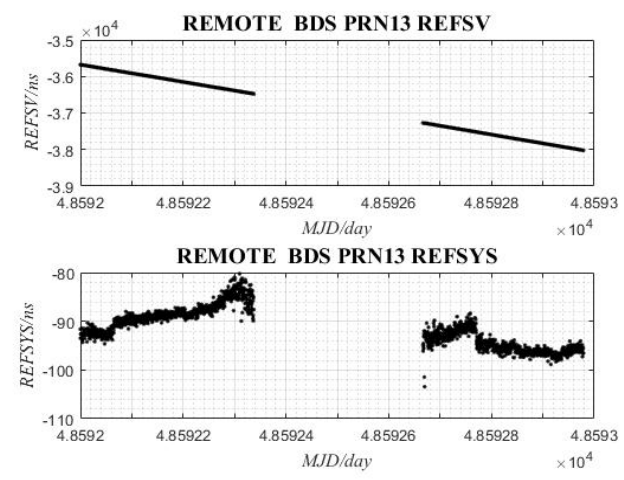

FIGURE V. THE REFSV AND REFSYS AT REMOTE STATION BASED PRN13 OF BDS

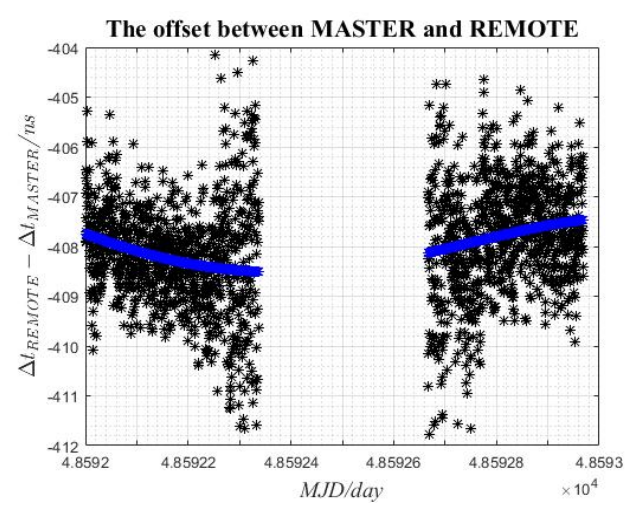

FIGURE VI. THE OFFSET BETWEEN THE LOCAL CLOCK AT MASTR STATION AND THE LOCAL CLOCK AT REMOTE STATION

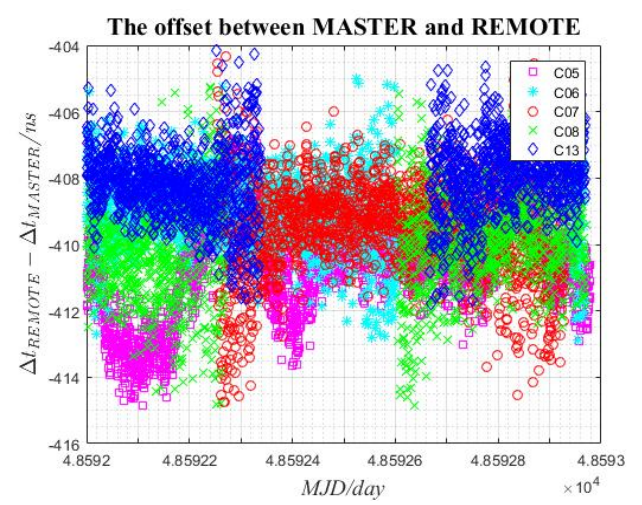

FIGURE VII. THE OFFSET BETWEEN THE LOCAL CLOCK AT MASTR STATION AND THE LOCAL CLOCK AT REMOTE STATION BASED ON MULTIPLE SATELLITES

TABLE I. THE OFFSET BETWEEN THE LOCAL CLOCK AT MASTR STATION AND THE LOCAL CLOCK AT REMOTE STATION BASED ON MULTIPLE SATELLITES

\begin{tabular}{ccc}
\hline Satellite ID & $\begin{array}{c}\text { std(ns) (least } \\
\text { square fitting) }\end{array}$ & $\begin{array}{c}\Delta t_{\text {REMOTE }}-\Delta t_{\text {MASTER }} \text { (ns) } \\
\text { (least square fitting) }\end{array}$ \\
\hline PRN05 & 0.743 & -410.598 \\
PRN06 & 0.249 & -408.951 \\
PRN07 & 0.404 & -409.520 \\
PRN08 & 0.309 & -410.007 \\
PRN13 & 0.311 & -408.008
\end{tabular}

As in FIGURE 7 and TABLE 1, there are five satellites in the data processing, including a GEO satellite identified PRN05. The difference of the local clock at REMOTE station and local clock at MASTER is about $-409 \mathrm{ns,} \mathrm{the} \mathrm{standard}$ deviation after least square fitting is less than $1 \mathrm{~ns}$ that is the accuracy less than $1 \mathrm{~ns}$ from TABLE 1 . But the standard deviation of the PRN05 is greater than others, because the lower elevation of the PRN05, and the signals could therefore suffer from more noise and multipath.

\section{CONCLUSIONS}

The paper has been realized the common-view based on BDS according to the standard of CGGTTS V2E. These results in very short baseline shows that the IGSO increases the BeiDou satellite availability, though it will bring a bit more noise into the result. However, the CV results obtained from GEO satellite are significantly noisier.

\section{REFERENCES}

[1] Allan, Thomas. Technical Directives for Standardization of GPS Time Receiver Software [J]. Metrologia, 1994(31):69-79.

[2] Lewandowski W, Azoubib J, Gevorkyan A G, et al. A Contribution to the Standardization of GPS and GLONASS Time Transfers: PTTI, Virgiuia, 1996 [C].

[3] Defraigne, Petit. CGGTTS-Version 2E: an extended standard for GNSS Time Transfer [J]. Metrologia, 2015.

[4] Wei-Peng L I, Jian-Wen L I, Dai W. Study on the methods of updating Klobuchar ionospheric delay correction model $[\mathrm{J}]$. Science of Surveying \& Mapping, 2009, 34(5):49-51.

[5] Boehm, Niell, Tregoning, et al. Global Mapping Function (GMF): A new empirical mapping function based on numerical weather model data [J]. Geophysical Research Letters, 2006. 
[6] BeiDou Navigation Satellite System Signal In Space Interface Control Document Open Service Signal (Version 2.1) [R].China Satellite Navigation Office, 2016.

[7] Teunissen Peter.J.G, Montenbruck O. Handbook of Global Navigation Satellite Systems [M]. Springer, 2017. 